\title{
In-Situ TEM Studies of the Deformation Mechanism of Nanocrystaline Ni
}

\author{
Zhiwei Shan ${ }^{1}$, Eric A. Stach ${ }^{2,5}$, Joerg M.K. Wiezorek ${ }^{3}$, David M. Follstaedt ${ }^{4}$, James A. \\ Knapp ${ }^{4}$, Scott X. Mao ${ }^{1}$ \\ 1 Department of Mechanical Engineering, University of Pittsburgh, Pittsburgh, PA \\ 15261, USA \\ 2 National Center for Electron Microscopy, Lawrence Berkeley National Laboratory, \\ Berkeley, CA 94720 \\ 3 Department of Materials Science and Engineering, University of Pittsburgh, \\ Pittsburgh, PA 15261, USA
}

4. Sandia National Laboratories, Albuquerque, NM 87185, USA

5. School of Materials Engineering, Purdue University, West Lafayette, In 47907

Nanocrystalline metals exhibit remarkable mechanical properties relative to their coarsegrained counterparts, including high strength, high hardness and an enhanced ability to deform superplastically. Despite this fact, an understanding of the physical mechanisms responsible for these basic properties remains elusive. Based on abundant indirect evidence and physical insight from molecular dynamics simulations, it has been largely accepted that with the decrease of grain size, a crossover regime exists, where the deformation mechanism of nanocrystalline materials will change continuously from dislocation nucleation and motion to grain boundary (GB) mediated plasticity, i.e. from an intragranular process to an intergranular one. However, to date no conclusive direct experimental evidence for the operation of non-dislocation based plasticity mechanism has been detected.

Here we show by solving the challenging problems encountered in previous studies, in situ dynamics dark-field transmission electron microscopy (DFTEM) has been done successfully which reveal conclusive experimental evidence that GB mediated plasticity, such as grain boundary sliding and grain rotation, become a prominent deformation mode for as-deposited $\mathrm{Ni}$ with an average grain size of about $10 \mathrm{~nm}$ [Fig. 1]. Moreover, no deformation twins were found in grains that are still in a strained state. Theoretical analysis suggested that the deformation mechanism crossover resulted from the competition between the deformation controlled by nucleation and motion of dislocations and the deformation controlled by GB related deformation accommodated mainly by GB diffusion with decreasing grain size $[1,2]$

We have also used nano-beam electron diffraction in the TEM to study the behavior of individual grains in nanocrystalline $\mathrm{Ni}$ during deformation under low local strain rate conditions. Direct measurement of lattice distortions during straining reveals that grain interiors may experience ultra-high elastic distortions during tensile deformation. These observations allow us to critically evaluate two highly cited models, which predict a critical grain size below which dislocation pile up or multiplication cease operation. We find that only the dislocation multiplication model can reasonably predict this critical grain size. Based on our application on dislocation multiplication model, we find the critical grain size in nickel to lie between $3.4 \sim 10.5 \mathrm{~nm}$, depending on the type of dislocation [3]. This agrees with our observations surprisingly well [4]. 
[1]. Shan, Z.W., et al., Grain boundary-mediated plasticity in nanocrystalline nickel. Science, 2004. 305: p. 654-657.

[2]. Shan, Z.W. and S.X. Mao, Direct evidence of a deformation mechanism crossover in nanocrystalline nickel. Accepted by Advanced Engineering Materials, 2005.

[3]. Shan, Z.W., et al., Ultrahigh elastic deformability of individual grains in nanocrystalline Ni. Submitted to Physical Review Letters.

[4]. Shan, Z.W., et al., Dislocation dynamics in nanocrystalline Ni. In preparation.

[5]. This work was supported by the US NSF grant CMS-0140317 to the University of Pittsburgh. The work at Sandia was supported by the Division of Materials Sciences and Engineering, Office of Basic Energy Sciences, of the U.S. Department of Energy. Sandia is a multiprogram laboratory operated by Sandia Corporation, a Lockheed Martin Company, for the United States Department of Energy's National Nuclear Security Administration under contract DE-AC0494AL85000.

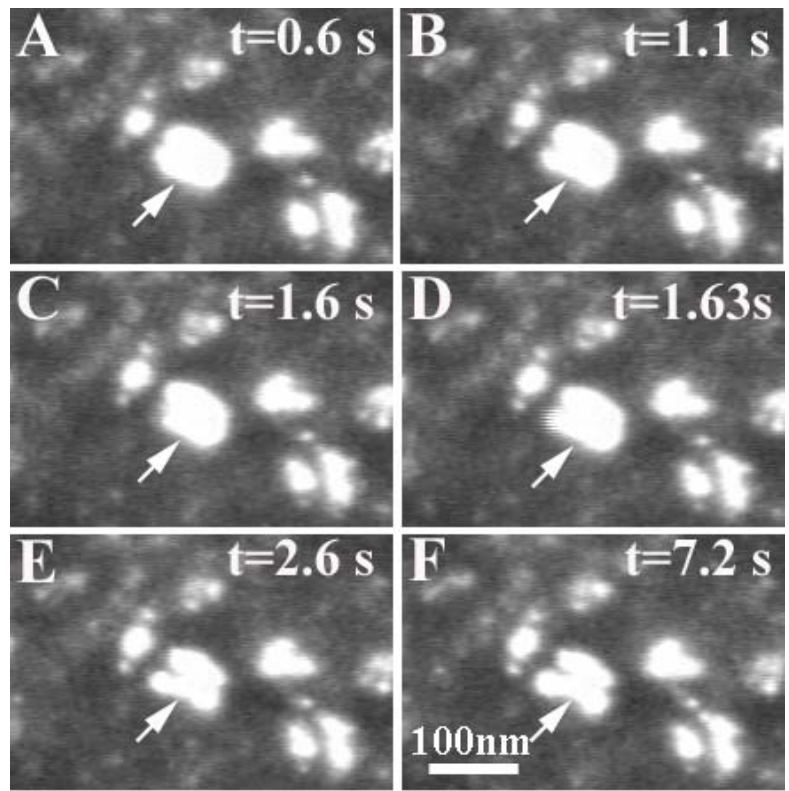

Figure1. DFTEM observation depicted the evolution of the newly grown agglomerate immediately following the image sequence shown in figure 3 of reference [1]. (A) $t=0.6 \mathrm{~s}$; (B) $t=1.1 \mathrm{~s}$, a small notch begins to emerge on the middle left of the agglomerate. (C) $t=1.6 \mathrm{~s}$, the lower left corner of the agglomerate lost its contrast gradually. (D) $t=1.63 \mathrm{~s}$, the lower left corner presented itself again in a sudden manner, which indicates sudden grain rotation. (E) $t=2.6 \mathrm{~s}$, the agglomerate appears to split into two elongated parts. (F) $\mathrm{t}=7.2 \mathrm{~s}$, the evolution of the agglomerate has all but stopped since $\mathrm{t}=2.6 \mathrm{~s}$, as a state of equilibrium is approached. 